\title{
Ultra-low contrast percutaneous coronary intervention in patients with severe chronic kidney disease
}

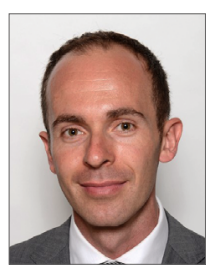

Lorenzo Azzalini*, MD, PhD, MSc; Daisuke Hachinohe, MD; Damiano Regazzoli, MD; Antonio Colombo, MD

Division of Interventional Cardiology, Cardio-Thoracic-Vascular Department, San Raffaele Scientific Institute, Milan, Italy This paper also includes supplementary data published online at: http://www.pcronline.com/eurointervention/141st_issue/160
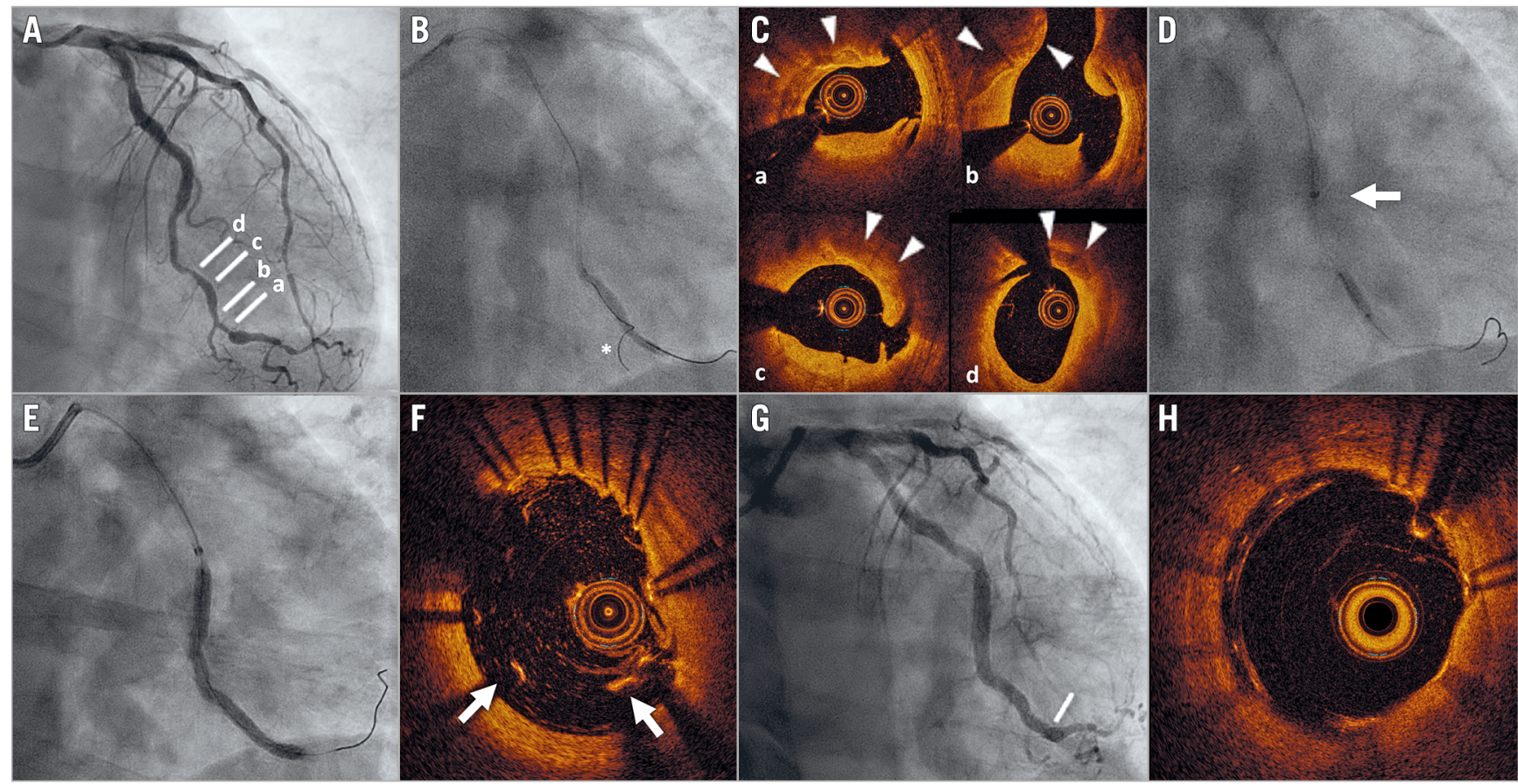

Figure 1. Ultra-low contrast PCI. A) Coronary angiography demonstrates a long and tortuous lesion in the distal circumflex. B) Predilatation with $2.5 \times 30 \mathrm{~mm}$ semi-compliant balloon. The asterisk indicates the wire advanced into a small side branch and used to create a "roadmap" for PCI. C) OCT shows extensive calcification (arrowheads) and multiple dissection planes (cross-sections at the levels indicated in panel A). D) Atherectomy with $2.5 \mathrm{~mm}$ scoring balloon (arrow indicates mother-and-child catheter). E) Implantation of 3.5-3.0 $\times 50 \mathrm{~mm}$ sirolimuseluting stent. F) OCT imaging shows major malapposition (arrows) of the stent at the distal edge (corresponding to the level indicated in panel $G)$. Good final angiographic $(G)$ and $O C T(H)$ result after repeat high-pressure post-dilatation.

*Corresponding author: Division of Interventional Cardiology, Cardio-Thoracic-Vascular Department, San Raffaele Scientific Institute, Via Olgettina 60, 20132 Milan, Italy.E-mail: azzalini.lorenzo@hsr.it 
A 52-year-old man with a prior kidney transplant and chronic kidney disease (CKD) (baseline creatinine $2.7 \mathrm{mg} / \mathrm{dl}$; estimated glomerular filtration rate [eGFR] $25 \mathrm{ml} / \mathrm{min} / 1.73 \mathrm{~m}^{2}$ ) presented with angina. Coronary angiography demonstrated two-vessel disease, with a long and tortuous lesion in the distal circumflex and diffuse disease in the distal left anterior descending, which was not considered to be amenable to treatment (Figure 1A, Moving image 1). The patient was referred for percutaneous coronary intervention (PCI) on the circumflex.

Our ultra-low contrast (ULC) PCI protocol is used in patients with eGFR $<30 \mathrm{ml} / \mathrm{min} / 1.73 \mathrm{~m}^{2}$ and comprises the use of: 1 ) ioversol, a low-osmolar contrast medium, diluted at $50 \%$, and used only in key moments of the procedure (e.g., to confirm the final result); 2) side branch wiring to create a "roadmap" for PCI; 3) optical coherence tomography (OCT) with dextran 40 (instead of contrast) to guide PCI (flow rate: $4.0 \mathrm{ml} / \mathrm{s}$, total volume: $14.0 \mathrm{ml}$, pressure: 400 psi, rise time: $0.0 \mathrm{~s}$ ). Additionally, patients receive hydration with isotonic saline $(1.5 \mathrm{ml} / \mathrm{kg} / \mathrm{hr}$ for 12 hours before and 24 hours after PCI).

A 6 Fr XB 3.5 guiding catheter was inserted transradially to engage the left main. Two wires were placed in the distal circumflex and one of its branches. After a small balloon was advanced with difficulty (due to marked tortuosity) and predilatation was performed (Figure 1B), a mother-and-child catheter was advanced into the distal circumflex. Dextran-based OCT was performed and revealed extensive calcification (Figure 1C, Moving image 2). Scoring balloon angioplasty was performed (Figure 1D), and a 3.5$3.0 \times 50 \mathrm{~mm}$ sirolimus-eluting stent (BioMime Morph ${ }^{\mathrm{TM}}$; Meril Life Sciences Pvt., Vapi, India) was implanted (Figure 1E) and post-dilated. Repeat OCT showed major stent malapposition at the distal edge (Figure 1F), which was resolved with repeat highpressure post-dilatation. Final angiographic (Figure 1G, Moving image 3) and OCT (Figure 1H, Moving image 4) results were good. Contrast volume was $12.5 \mathrm{ml}$, fluoroscopy time $35 \mathrm{~min}$, and radiation dose $65 \mathrm{~Gy} \cdot \mathrm{cm}^{2}$. Creatinine at 48 and 72 hours remained at $2.7 \mathrm{mg} / \mathrm{dl}$. At 30-day follow-up, the patient was asymptomatic and had not suffered any hospitalisation.

The incidence of contrast-induced nephropathy (CIN) in patients with severe CKD is high $(26.6 \%)$, and its consequences devastating, including a high risk of in-hospital mortality $(9.7 \%)^{1}$. CKD subjects undergo PCI less frequently than non-CKD patients, due to aversion to the risk of CIN. In such a setting, the implementation of ULC-PCI protocols is warranted. Diluted contrast is a feasible option to reduce contrast volume in PCI while maintaining sufficient image quality ${ }^{2}$. Dextran has been shown to be effective and safe for blood flushing during $\mathrm{OCT}^{3}$. Zero-contrast ${ }^{4}$ and lowcontrast $^{2}$ PCI protocols based on intravascular ultrasound (IVUS) have the potential to decrease the incidence of CIN, although IVUS significantly prolongs these procedures and has lower resolution compared with OCT. Additionally, a zero-contrast approach might prevent the prompt diagnosis of life-threatening complications, such as distal-vessel perforation. Therefore, ULC-PCI based on diluted contrast and dextran-based OCT appears to be an appealing option in patients with advanced CKD and deserves further investigation.

\section{Conflict of interest statement}

L. Azzalini reports grants from ACIST Medical Systems, and personal fees from Guerbet, outside the submitted work. The other authors have no conflicts of interest to declare.

\section{References}

1. Tsai TT, Patel UD, Chang TI, Kennedy KF, Masoudi FA, Matheny ME, Kosiborod M, Amin AP, Messenger JC, Rumsfeld JS, Spertus JA. Contemporary incidence, predictors, and outcomes of acute kidney injury in patients undergoing percutaneous coronary interventions: insights from the NCDR Cath-PCI registry. JACC Cardiovasc Interv. 2014;7:1-9.

2. Mariani J Jr, Guedes C, Soares P, Zalc S, Campos CM, Lopes AC, Spadaro AG, Perin MA, Esteves Filho A, Takimura CK, Ribeiro E, Kalil-Filho R, Edelman ER, Serruys PW, Lemos PA. Intravascular ultrasound guidance to minimize the use of iodine contrast in percutaneous coronary intervention: the MOZART (Minimizing cOntrast utiliZation With IVUS Guidance in coRonary angioplasTy) randomized controlled trial. JACC Cardiovasc Interv. 2014;7:1287-93.

3. Frick K, Michael TT, Alomar M, Mohammed A, Rangan BV, Abdullah S, Grodin J, Hastings JL, Banerjee S, Brilakis ES. Low molecular weight dextran provides similar optical coherence tomography coronary imaging compared to radiographic contrast media. Catheter Cardiovasc Interv. 2014;84:727-31.

4. Ali ZA, Karimi Galougahi K, Nazif T, Maehara A, Hardy MA, Cohen DJ, Ratner LE, Collins MB, Moses JW, Kirtane AJ, Stone GW, Karmpaliotis D, Leon MB. Imaging- and physiologyguided percutaneous coronary intervention without contrast administration in advanced renal failure: a feasibility, safety, and outcome study. Eur Heart J. 2016;37:3090-5.

\section{Supplementary data}

Moving image 1. Baseline coronary angiogram (non-diluted contrast). Moving image 2. Dextran-based optical coherence tomography after predilatation.

Moving image 3. Final angiographic result (diluted contrast).

Moving image 4. Final dextran-based optical coherence tomography.

The supplementary data are published online at:

http://www.pcronline.com/

eurointervention/141st issue/160 